\title{
RF-based Direction Finding of UAVs Using DNN
}

\author{
Samith Abeywickrama*†, Lahiru Jayasinghe ${ }^{\dagger}$, Hua Fu ${ }^{\dagger}$, Subashini Nissanka ${ }^{\dagger}$, and Chau Yuen ${ }^{\dagger}$ \\ ${ }^{\dagger}$ Singapore University of Technology and Design, Singapore \\ ${ }^{*}$ National University of Singapore, Singapore \\ Email: samith@u.nus.edu, \{aruna_jayasinghe,hua_fu,yuenchau\}@sutd.edu.sg
}

\begin{abstract}
This paper presents a sparse denoising autoencoder (SDAE)-based deep neural network (DNN) for the direction finding (DF) of small unmanned aerial vehicles (UAVs). It is motivated by the practical challenges associated with classical DF algorithms such as MUSIC and ESPRIT. The proposed DF scheme is practical and low-complex in the sense that a phase synchronization mechanism, an antenna calibration mechanism, and the analytical model of the antenna radiation pattern are not essential. Also, the proposed DF method can be implemented using a single-channel RF receiver. The paper validates the proposed method experimentally as well.
\end{abstract}

Index Terms-Drone surveillance, direction finding, UAV tracking

\section{INTRODUCTION}

The use of civilian drones has increased dramatically in recent years. Likewise, drones are fast gaining popularity around the world [1-5]. However, drone use in a problematic manner has stirred public concerns. For example, in January 2015 a drone crashed at the White House [6], raising concerns about security risks to the government building; in March 2016 a Lufthansa jet came within 200 feet of colliding with a drone near Los Angeles International Airport [7]; and drones have been accused of being used to violate the privacy and even carry criminal activities [8]. These events give ample selfevident examples that developing a surveillance system for suspect drones is of paramount importance.

The authors of [9] sought to detect a drone using Radio Frequency (RF) as it can work day and night and at all weather conditions. Most of the commercial drones communicate frequently with their controllers, and the downlink, i.e., video signal and telemetry signals (flight speed, position, altitude, and battery level), between the drone and its controller is always present. To this end, this paper presents a drone surveillance system, by eavesdropping on the communication between a drone and its ground controller. The system can estimate drone's direction (or bearing) by processing the data transmitted from the drone to its controller using a single channel wireless receiver. Therefore, no dedicated transmitter is required at the surveillance system.

RF based direction finding (DF) techniques have been well studied, and the classical high-resolution techniques such as

The support of NSFC 61750110529 and SUTD-MIT International Design Center is gratefully acknowledged. The first two authors contributed equally.
MUSIC [10] and ESPRIT [11] are considered to be the most popular algorithms. However, MUSIC and ESPRIT are inherently multi-channel techniques because those algorithms require a snapshot observation. This means, the base-band data from all antenna elements should be extracted simultaneously so that a data correlation matrix can be formulated. Therefore, multiple channels should be coherent. However, in most receivers, the digital down converter (DDC) chain uses a coordinate rotation digital computer (CORDIC), which has a random start-up position on power up. The CORDIC therefore creates a random phase each time when the channels of the receiver are initialized, but remains constant throughout the operation [12]. Therefore, calibrating this start-up phase values of each RF channel becomes necessary to realize a coherent multi-channel receiver. Clearly, this increases the hardware complexity and the power consumption.

Most of the civilian drones use WiFi-like OFDM for their communication. They are usually unknown, wideband, and transmitted in burst-mode. Such signal characteristics pose challenges with classical DF techniques. However, if only the signal power measurements are utilized, performing DF is practically feasible even with such signals [13-16]. In [16], signal power measurements that are obtained from a switched beam antenna array are utilized to estimate the direction of a WiFi transmitter. As the actual radiation pattern of the antenna is vital for these methods, still it bounds with some practical challenges. Therefore, we propose a practical and a lowcomplex drone DF method in this paper, and our contributions can be summarized as fallows.

To the best of authors' knowledge there has not been any other method that involves deep neural network in the context of drone DF. We focus on a system which comprises a directional antenna array having $N$ antennas, and a single channel receiver. By processing the signals that are transmitted from the drone to its ground controller, the single channel receiver measures the received signal power at the each antenna using a RF switching mechanism. Then, the obtained power values are fed to the proposed sparse denoising autoencoder (SDAE)-based deep neural network (DNN). More precisely, the first hidden layer of the network extracts a robust sparse representation of the received power values. Then, the rest of the network utilizes this sparse representation to classify the direction of the drone signal. It should be noted that a phase synchronization mechanism, 


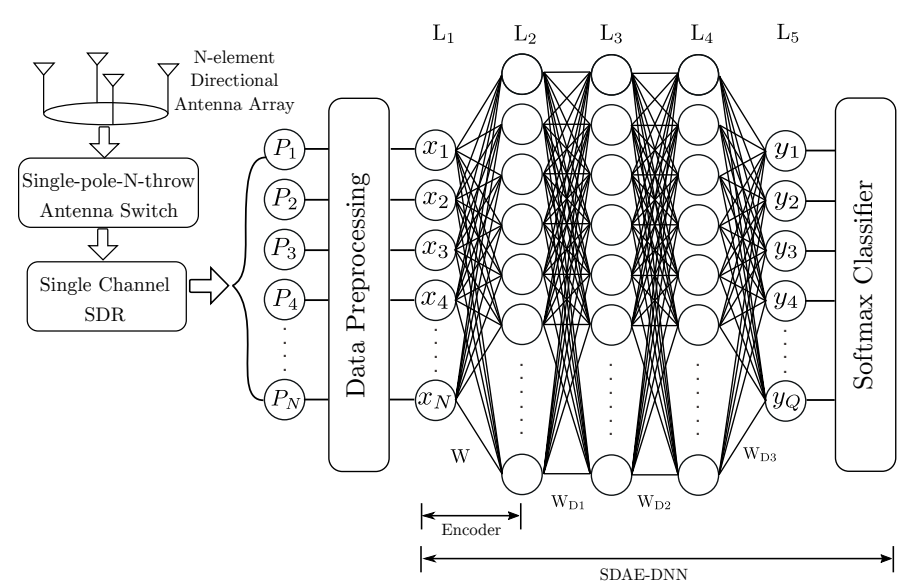

Fig. 1. The System Model.

an antenna gain calibration mechanism, and the analytical model of the antenna radiation pattern are not essential for this single channel implementation. The paper validates the proposed method experimentally through a software defined radio (SDR) implementation in conjunction with TensorFlow [17]. Furthermore, such an experimental validation for drone DF is not common in the literature, and can be highlighted as another contribution of this paper.

The paper organization is as follows. The system model is presented in Section [I. Section [II discusses the proposed deep architecture. Then, in Section IV, we validate the proposed method using experimental results. Section $\nabla$ concludes the paper.

To promote reproducible research, the codes for generating most of the results in the paper are made available on the website: https://github.com/LahiruJayasinghe/DeepDOA.

\section{SySTEM MODEL}

We consider a system which consists of a single channel receiver, and a circular antenna array equipped with $N$ direction antennas, see Fig. 1 The antenna array is connected to the receiver using a non-reflective Single-Pole-N-Throw (SPNT) $\mathrm{RF}$ switch. The switching period is $T_{s}$. Suppose that a farfield drone signal impinges on the antenna array with azimuth angle $\theta \in\left[\begin{array}{ll}0 & 2 \pi\end{array}\right)$. The received signal at the $n$-th antenna element can be given as

$$
r_{n}(k)=a_{n}(\theta) s(k)+n_{n}(k),
$$

where $k$ is the sample index, $a_{n}(\theta)$ is the $n$-th antenna response vector for the azimuth angle $\theta, s(k)$ is the drone transmitted signal as it arrives at the antenna array, $n_{n}(k)$ is circularly symmetric, independent and identically distributed, complex additive white Gaussian noise (AWGN) with zero mean and variance $\sigma^{2}$, and $n \in\{1, \cdots, N\}$. Here, $a_{n}(\theta)$ follows the form of

$$
a_{n}(\theta)=G_{n}(\theta) e^{j \frac{2 \pi}{\lambda} \beta_{n}(\theta)},
$$

where $G_{n}(\theta)$ is the real numbered antenna gain for the azimuth angle $\theta, \lambda$ is the signal wavelength, and

$$
\beta_{n}(\theta)=d \cos \left[\frac{2 \pi(n-1)}{N}-\theta\right],
$$

where $d$ is the radius of the circular antenna array [18]. Since we consider a practical DF method in this paper, $s(k)$ and $a_{n}(\theta)$ are assumed to be unknown. Therefore, our objective is to recover the azimuth angle $\theta$, while the parameters $s(k)$ and $a_{n}(\theta)$ are unknown.

We focus on a power measurements based approach. To this end, the ensemble averaged received signal power at the $n$-th antenna element can be given as

$$
\begin{aligned}
P_{n} & =\mathrm{E}\left[\left|r_{n}(k)\right|^{2}\right] \\
& =\mathrm{E}\left[\left(a_{n}(\theta) s(k)+n_{n}(k)\right)\left(a_{n}(\theta) s(k)+n_{n}(k)\right)^{*}\right] \\
& =\left|a_{n}(\theta)\right|^{2} \mathrm{E}\left[|s(k)|^{2}\right]+\mathrm{E}\left[\left|n_{n}(k)\right|^{2}\right] \\
& =G_{n}^{2}(\theta) P_{s}+\sigma^{2},
\end{aligned}
$$

where

$$
\begin{gathered}
G_{n}^{2}(\theta)=\left|a_{n}(\theta)\right|^{2}, \\
P_{s}=\mathrm{E}\left[|s(k)|^{2}\right], \\
\sigma^{2}=\mathrm{E}\left[\left|n_{n}(k)\right|^{2}\right],
\end{gathered}
$$

and $E[\cdot]$ denotes the expectation operator. Here, (4) follows from the fact that $s(k)$ and $n_{n}(k)$ are independent and uncorrelated, i.e.,

$$
\mathrm{E}\left[s(k) n_{n}^{*}(n)\right]=\mathrm{E}\left[s^{*}(k) n_{n}(n)\right]=0 .
$$

It can be observed that the received power values at the antenna elements $n_{i}$ and $n_{j}$, where $i, j \in\{1, \cdots, N\}$ and $i \neq j$, are not identical, since $G_{i}^{2}(\theta) \neq G_{j}^{2}(\theta)$. We have this property thanks to the gain variation of the directional antenna array in $\left[\begin{array}{ll}0 & 2 \pi\end{array}\right)$. Therefore, it is desirable to have an underlying relationship (or a pattern) between $\left\{P_{n}\right\}_{n=1}^{N}$ and $\theta$.

The proposed method is as follows. The receiver sequentially activates one antenna element at a time using the SPNT RF switch, and measures the corresponding received power value. During the activation of $n$-th antenna, $P_{n}$ is measured, where $n \in\{1, \cdots, N\}$. A single switching cycle is equivalent to $N$ activations, starting from the first antenna to the $N$-th antenna. Let $\mathbf{p}=\left[P_{1}, \cdots, P_{N}\right]^{\top}$ denote the power measurements corresponding to a single switching cycle. As it is depicted in Fig. 1, $\boldsymbol{x}=\left[x_{1}, \cdots, x_{N}\right]^{\top}$ is obtained during the preprocessing stage, where

$$
x_{n}=\frac{P_{n}}{\sum_{i=1}^{N} P_{i}} .
$$

This means, $x_{n}$ is the ratio between $P_{n}$ and the summation of all power values within the same switching cycle. In the next section, we discuss how the proposed network recovers $\theta$ from $\boldsymbol{x}$. 


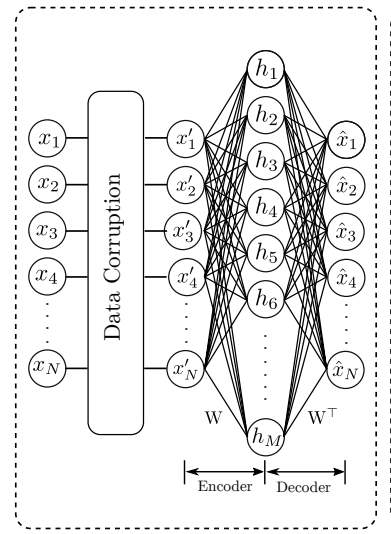

(a) SDAE Training Phase

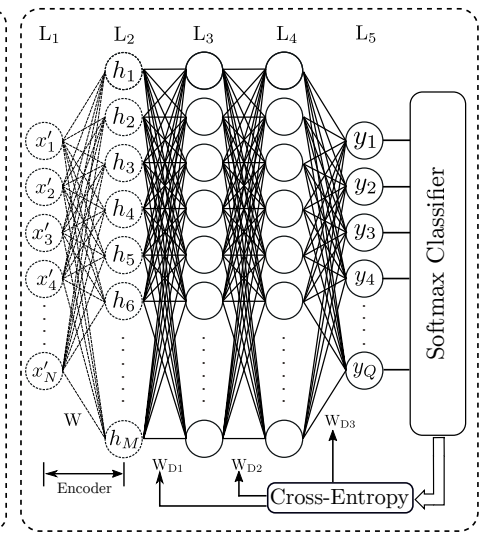

(b) DNN Training Phase
Fig. 2. Training Phases.

\section{SDAE-DNN ARCHITECTURE}

The proposed deep architecture comprises a trained SDAE and a trained DNN, followed by a fully-connected softmax classifier layer, see Fig. 1 During the training phase of the SDAE (Fig. 2-(a)), the preprocessed received power values $\left\{x_{n}\right\}_{n=1}^{N}$ are assigned to the input units. Therefore, the number of neurons in the input layer is equal to the number of elements $N$ in the directional antenna array. Then, the values of the hidden layer units are calculated as

$$
\boldsymbol{h}=f\left(\mathbf{W} f_{c}(\boldsymbol{x})+\boldsymbol{b}_{\boldsymbol{e}}\right),
$$

and output layer values are calculated as

$$
\hat{\boldsymbol{x}}=f\left(\mathbf{W}^{\top} \boldsymbol{h}+\boldsymbol{b}_{\boldsymbol{d}}\right),
$$

where $f(\cdot)$ is non-linear activation function that operates element-wise on its argument, $\mathbf{W} \in \mathbb{R}^{M \times N}$ denotes the encoder weight matrix, $\boldsymbol{b}_{\boldsymbol{e}}=\left[b_{e_{1}}, \ldots, b_{e_{M}}\right]^{\top}$ and $\boldsymbol{b}_{\boldsymbol{d}}=$ $\left[b_{d_{1}}, \ldots, b_{d_{N}}\right]^{\top}$ denote the bias vectors, and $f_{c}(\cdot)$ is a stochastic corrupter which adds noise according to some noise model to its input, i.e., $\boldsymbol{x}^{\prime}=f_{c}(\boldsymbol{x})$, where $\boldsymbol{x}^{\prime}=\left[x_{1}^{\prime}, \ldots, x_{N}^{\prime}\right]^{\top}$. In (6), $f_{c}$ is non-deterministic, since it corrupts the same set of received power values $\left\{x_{n}\right\}_{n=1}^{N}$ in different ways every time $\left\{x_{n}\right\}_{n=1}^{N}$ is passed through it. $\mathbf{W}^{\top}$ is the decoder weight matrix, which ensures that the output layer reconstructs the input as precisely as possible $\left(\mathbf{W}^{\top}\right.$ is the matrix transpose of W). Here, we particularly target on reconstructing the input received power values at the output layer of the SDAE.

To this end, the parameters of $\operatorname{SDAE}\left(\mathbf{W}, \boldsymbol{b}_{\boldsymbol{e}}\right.$, and $\left.\boldsymbol{b}_{\boldsymbol{d}}\right)$ are optimized such that the reconstruction error is minimized, while it subjecting to a sparsity constraint. This sparsity constraint encourages the sparse activation of the hidden layer units. Therefore, the cost function can be given as

$$
L\left(\mathbf{W}, \boldsymbol{b}_{\boldsymbol{e}}, \boldsymbol{b}_{\boldsymbol{d}}\right)=\sum_{i=1}^{T}\left(\hat{\boldsymbol{x}}_{\boldsymbol{i}}-\boldsymbol{x}_{\boldsymbol{i}}\right)^{2}+\beta \sum_{m=1}^{M} \mathrm{KL}\left(\rho \| \rho_{m}\right),
$$

where $T$ is the size of the training data set, $\beta$ is a hyper parameter 1 , $\rho$ is the sparsity parameter,

$$
\rho_{m}=\frac{1}{T} \sum_{i=1}^{T} h_{m}\left(\boldsymbol{x}_{\boldsymbol{i}}\right)
$$

is the average activation level of the $m$-th hidden unit where $h_{m}\left(\boldsymbol{x}_{\boldsymbol{i}}\right)$ denotes the activation of the $m$-th unit for the input $\boldsymbol{x}_{\boldsymbol{i}}$, and

$$
\mathrm{KL}\left(\rho \| \rho_{m}\right)=\rho \log \left(\frac{\rho}{\rho_{m}}\right)+(1-\rho) \log \left(\frac{1-\rho}{1-\rho_{m}}\right)
$$

is the Kullback-Leibler (KL) divergence [19]. From (9), it can be observed that $\operatorname{KL}\left(\rho \| \rho_{m}\right)=0$, if $\rho_{m}=\rho$, and otherwise it increases monotonically as $\rho_{m}$ diverges from $\rho$. Typically, $\rho$ is a very small value close to zero. Therefore, when the cost function (8) is minimized, the parameter $\rho$ enforces $\left\{\rho_{m}\right\}_{m=1}^{M}$ to be close to zero, while the dominant neurons that represent specific features stay non-zero. Now, the decoder is discarded, and the trained encoder is connected to the DNN as a fully connected layer.

Next, the DNN training phase is commenced. As Fig. 2. (b) depicts, DNN comprises three fully connected hidden layers, i.e., $\mathrm{L}_{2}, \mathrm{~L}_{3}$, and $\mathrm{L}_{4}$, and a softmax layer [20] for the task of classification. Since $\mathrm{L}_{2}$ is an element of the trained encoder, it uses the same activation function $f$. Hidden layers $\mathrm{L}_{3}$ and $\mathrm{L}_{4}$ use Rectified liner Unit (ReLU) [21-23] as their activation function. Again, noise corrupted received power values $\left\{x_{n}^{\prime}\right\}_{n=1}^{N}$ are the training inputs. Now, data need to be labelled into $Q$ classes due to the use of softmax classifier, where the label is the direction of the drone signal coming from. Therefore, the learning strategy is supervised in this training phase. Since, $\mathbf{W}$ is the pre-trained encoder weight matrix, it will not be optimized again. Therefore, only the weight matrices $\mathbf{W}_{\mathrm{D} 1}, \mathbf{W}_{\mathrm{D} 2}$, and $\mathbf{W}_{\mathrm{D} 3}$ are optimized during this training phase.

Remark 1: It should be noted that the incoming drone signal with direction $\theta$ occupies a certain isolated point in the angle domain of $\left[\begin{array}{ll}0 & 2 \pi\end{array}\right)$. Therefore, $\theta$ is sparse in the spatial domain, and this sparsity can be exploited to estimate $\theta$. Here, we use this sparse property, and it can be summarized as follows. In the cost function (8), the squared error is calculated between the non-corrupted power values and the reconstructed power values, while the noise-corrupted power values are fed to the network. This cost function is subject to a sparsity constraint as well. Therefore, even when the system operates in a noisy environment, the first hidden layer (or $\mathbf{W}$ ) of the network extracts a robust sparse representation of the input power values. Then, the rest of the network utilizes this sparse representation to classify (or estimate) $\theta$.

In the next section, we will validate our proposed method using experimental results.

$1 \beta$ operates as the trade-off parameter between the squared error and $\operatorname{KL}\left(\rho \| \rho_{m}\right)$, and its value can be empirically decided during the training process. 
TABLE I

CONFUSION MATRIX WHEN THE PROPOSED NETWORK IS USED.

\begin{tabular}{|c|c|c|c|c|c|c|c|c|}
\cline { 2 - 9 } \multicolumn{1}{c|}{} & $0^{0}$ & $45^{0}$ & $90^{0}$ & $135^{0}$ & $180^{0}$ & $225^{0}$ & $270^{0}$ & $315^{0}$ \\
\hline $0^{0}$ & $\mathbf{9 5}$ & 1 & 1 & 0 & 3 & 0 & 0 & 0 \\
\hline $45^{0}$ & 1 & $\mathbf{9 7}$ & 0 & 0 & 1 & 1 & 0 & 0 \\
\hline $90^{0}$ & 1 & 1 & $\mathbf{9 8}$ & 0 & 0 & 0 & 0 & 0 \\
\hline $135^{0}$ & 0 & 0 & 0 & $\mathbf{1 0 0}$ & 0 & 0 & 0 & 0 \\
\hline $180^{0}$ & 3 & 3 & 0 & 0 & $\mathbf{9 2}$ & 2 & 0 & 0 \\
\hline $225^{0}$ & 0 & 0 & 4 & 0 & 1 & $\mathbf{9 5}$ & 0 & 0 \\
\hline $270^{0}$ & 0 & 0 & 3 & 0 & 0 & 1 & $\mathbf{9 5}$ & 1 \\
\hline $315^{0}$ & 0 & 0 & 0 & 0 & 0 & 0 & 1 & $\mathbf{9 9}$ \\
\hline
\end{tabular}

\section{EXPERIMENTAL VALIDATION}

Our experimental setup comprises a SDR (USRP B210), and a four element sector antenna, which is a variant of the antenna implemented in [24]. We use only a single RF receiving channel of the SDR. Therefore, the SDR is connected to the antenna using a non-reflective Single-Pole-4Throw (SP4T) RF switch. DJI Phantom 3 is considered as the target drone throughout the experiment. The drone downlink channels occupy the bandwidth from $2.401 \mathrm{GHz}$ to $2.481 \mathrm{GHz}$, each has $10 \mathrm{MHz}$ bandwidth OFDM signal. This OFDM signal transmitted by the drone provides the main source to perform the DF task.

Fig. 3-(a) represents the environment that we used for the training data collection. This is a large ground with an open area. Also, there was negligible RF interference on the $2.401 \mathrm{GHz}-2.481 \mathrm{GHz}$ range. To simplify the experiment, we virtually divided the area into eight octants, see Fig. 3. (b). Each octant is considered as one direction during the experiment. For example, the first octant is considered as 0 degrees direction, while the second octant is considered as 45 degrees direction, and so on. Therefore, when the drone is flying, its direction is indicated by its corresponding octant.

In the trained network, $\mathrm{L}_{5}-$ th layer has eight neurons (we have eight classes for the direction classification, or, $Q=8$ ) and $\mathrm{L}_{1}$-th layer has four neurons (the antenna array has four elements, or, $N=4$ ). The hidden layers $\mathrm{L}_{2}, \mathrm{~L}_{3}$, and $\mathrm{L}_{4}$ have 200,12 , and 12 neurons, respectively. These values have been empirically decided during the training process.

After the training phase, the evaluation is done in a different environment. Now, the frequency spectrum $(2.401 \mathrm{GHz}-$ $2.481 \mathrm{GHz}$ ) suffers from $\mathrm{WiFi}$ and bluetooth interferences. To this end, two experiments have been carried out. First, we evaluated the proposed deep architecture, and its confusion matrix is given in the Table I. Next, we considered a baseline method, where only a conventional DNN is implemented without the $\mathrm{L}_{2}$ layer (other layers have same number of nodes). Its confusion matrix is given in the Table II. Note that the confusion matries represent the percentage (\%) values. It can be observed that the proposed deep architecture is certainly
TABLE II

CONFUSION MATRIX WHEN ONLY THE DNN IS USED.

\begin{tabular}{|c|c|c|c|c|c|c|c|c|}
\cline { 2 - 9 } \multicolumn{1}{c|}{} & $0^{0}$ & $45^{0}$ & $90^{0}$ & $135^{0}$ & $180^{0}$ & $225^{0}$ & $270^{0}$ & $315^{0}$ \\
\hline $0^{0}$ & $\mathbf{9 4}$ & 5 & 1 & 0 & 0 & 0 & 0 & 0 \\
\hline $45^{0}$ & 10 & $\mathbf{8 8}$ & 1 & 0 & 0 & 1 & 0 & 0 \\
\hline $90^{0}$ & 4 & 1 & $\mathbf{8 8}$ & 1 & 3 & 0 & 0 & 3 \\
\hline $135^{0}$ & 0 & 6 & 1 & $\mathbf{9 3}$ & 0 & 0 & 0 & 0 \\
\hline $180^{0}$ & 30 & 4 & 3 & 0 & $\mathbf{6 0}$ & 2 & 0 & 1 \\
\hline $225^{0}$ & 1 & 0 & 4 & 0 & 2 & $\mathbf{9 1}$ & 0 & 2 \\
\hline $270^{0}$ & 0 & 0 & 1 & 0 & 2 & 1 & $\mathbf{9 4}$ & 2 \\
\hline $315^{0}$ & 0 & 0 & 0 & 0 & 3 & 0 & 0 & $\mathbf{9 7}$ \\
\hline
\end{tabular}

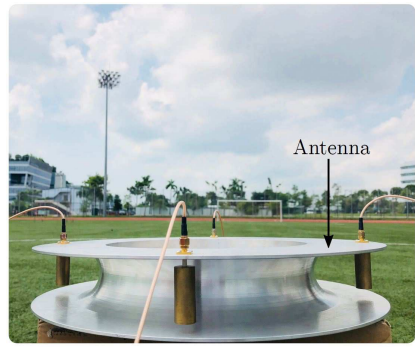

(a) Training Field

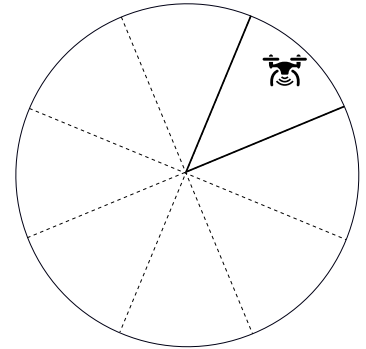

(b) Direction Configuration
Fig. 3. The Training Field and The Direction Configuration.

robust, and it outperforms the baseline method.

Since our implementation does not use multiple RF channels and any information about the antenna radiation pattern, it is not desirable to compare our results with conventional techniques. Therefore, we omit such simulation/experimental results. Further interesting experimental evaluations and insights will be presented in future extensions of this work.

\section{Conclusion}

This paper has proposed a novel DF method to be used in a drone surveillance system. The system comprises a single channel receiver and a directional antenna array. The receiver sequentially activates each antenna in the array, and measures the received power values. The power measurements corresponding to each switching cycle are fed to the proposed deep network. Then, it performs DF by exploiting the sparsity property of the incoming drone signal, and the gain variation property of the directional antenna array. The paper has validated the proposed method experimentally. Also, it has been proven that a phase synchronization mechanism, an antenna gain calibration mechanism, and the analytical model of the antenna radiation pattern are not essential for this single channel implementation. In future the scheme will be applied to portable, SDR-based prototype design [9] and field test and experiment. 


\section{REFERENCES}

[1] Y. Sun, H. Fu, S. Abeywickrama, L. Jayasinghe, C. Yuen, and J. Chen, "Drone classification and localization using micro-doppler signature with low-frequency signal," To appear in IEEE International Conference on Communication Systems, 2018.

[2] Q. Wu, Y. Zeng, and R. Zhang, "Joint trajectory and communication design for multi-UAV enabled wireless networks," IEEE Trans. Wireless Commun., vol. 17, no. 3, pp. 2109-2121, 2018.

[3] Q. Wu and R. Zhang, "Common throughput maximization in UAVenabled OFDMA systems with delay consideration," To appear in IEEE Trans. Commun., 2018.

[4] Q. Wu, J. Xu, and R. Zhang, "Capacity characterization of UAV-enabled two-user broadcast channel," To appear in IEEE J. Sel. Areas Commun., 2018.

[5] Q. Wu, L. Liu, and R. Zhang, "Fundamental tradeoffs in communication and trajectory design for UAV-enabled wireless network," Submitted to IEEE Trans. Wireless Commun., 2018.

[6] B. Jansen, "Drone crash at white house reveals security risks." [online] https://www.usatoday.com/story/news/2015/01/26/, 2015. [Accessed on 26 November 2017].

[7] J. Serna, "Lufthansa jet and drone nearly collide near lax." [online] www.latimes.com/local/lanow/la-me-ln-drone-near-miss-lax-20160318 2016. [Accessed on 26 November 2017].

[8] A. Morrow, "Couple accuses neighbor of stalking with drone." [online] https://www.suasnews.com/2014/12/. 2014. [Accessed on 26 November 2017].

[9] H. Fu, S. Abeywickrama, L. Zhang, and C. Yuen, "Low complexity portable passive drone surveillance via SDR-based signal processing," IEEE Commun. Mag., vol. 56, pp. 112-118, Apr. 2018.

[10] R. Schmidt, "Multiple emitter location and signal parameter estimation," IEEE Trans. Antennas and Propagation, vol. 34, pp. 276-280, Mar. 1986.

[11] R. Ray and T. Kailath, "ESPRIT-estimation of signal parameters via rotational invariance techniques," IEEE Trans. Acoustics, Speech, Signal Processing, vol. 37, pp. 984-995, Jul. 1989.

[12] J. van der Merwe, J. Malan, F. Maasdorp, and W. D. Plessis, "Multichannel software defined radio experimental evaluation and analysis," in
Proc. Instituto Tecnologico de Aeronautica (ITA), Sep. 2014.

[13] H. Fu, S. Abeywickrama, and C. Yuen, "A robust phase-ambiguityimmune doa estimation scheme for antenna array," To appear in IEEE International Conference on Communication Systems, 2018.

[14] S. Maddio, M. Passafiume, A. Cidronali, and G. Manes, "A closed-form formula for RSSI-based doa estimation with switched beam antennas," in Proc. 2015 European Radar Conference, Sep. 2015.

[15] R. Pöhlmann, S. Zhang, T. Jost, and A. Dammann, "Power-Based Direction-of-Arrival Estimation Using a Single Multi-Mode Antenna," ArXiv e-prints, Jun. 2017.

[16] S. Maddio, M. Passafiume, A. Cidronali, and G. Manes, "A scalable distributed positioning system augmenting wifi technology," in Proc. IEEE International Conference on Indoor Positioning and Indoor Navigation, Oct. 2013.

[17] M. Abadi and A. Agarwal, "Tensorflow: Large-scale machine learning on heterogeneous distributed systems," ArXiv e-prints, Mar. 2016.

[18] B. R. Jackson, S. Rajan, B. Liao, and S. Wang, "Direction of arrival estimation using directive antennas in uniform circular arrays," IEEE Trans. Antennas and Propagation, vol. 63, pp. 736-747, Feb. 2015.

[19] S. Kullback and R. Leibler, "On information and sufficiency," The Annals of Mathematical Statistics, vol. 22, pp. 79-86, Mar. 1951.

[20] C. Bishop, Pattern Recognition and Machine Learning. Springer-Verlag, 2006.

[21] K. Hara, D. Saito, and H. Shouno, "Analysis of function of rectified linear unit used in deep learning," in Proc. IEEE International Joint Conference on Neural Networks, Jul. 2015.

[22] L. Jayasinghe, N. Wijerathne, and C. Yuen, "A deep learning approach for classification of cleanliness in restrooms," To appear in Proc. IEEE International Conference on Intelligent and Advanced Systems, Aug. 2018.

[23] L. Jayasinghe, T. Samarasinghe, C. Yuen, and S. S. Ge, "Temporal convolutional memory networks for remaining useful life estimation of industrial machinery," To appear in IEEE International Conference on Industrial Technology, 2019.

[24] L. Yang, Z. Shen, W. Wu, and J. D. Zhang, "A four-element array of wideband low-profile H-Plane horns," IEEE Trans. Vehicular Technology, vol. 64, pp. 4356-4359, Sep. 2015. 